\title{
RAPID PROTOTYPING BOOST IN RESEARCH AND DEVELOPMENT
}

\author{
Karolina Macúchová ${ }^{1}$, Milan Melichar ${ }^{1}$, Pavel Crha ${ }^{1}$ and Jan Heřmánek ${ }^{1}$ \\ ${ }^{1}$ HiLASE Centre, Institute of Physics CAS, Za Radnicí 828, \\ 25241 Dolní Břežany, Czech Republic \\ Email: karolina.macuchova@hilase.cz
}

\begin{abstract}
In this article we demonstrate benefits that rapid prototyping methods bring to a modern hi-tech laboratory. Research and development facilities, such as the Hi-LASE centre, handles a huge amount of custom components that needs to be fitted and adjusted on site.

The HiLASE centre focuses on innovative laser technologies. Due to fast speed of research we have to speed up the design process as well. Production method based on in-house fast and cheap 3D printing enables effective testing of custom design solutions and avoids time delays when compared to outsourced common subtractive manufacturing technology. For numerous special design applications the additive 3D printing methods offers manufacturing capabilities that cannot be reached otherwise.
\end{abstract}

Keywords: Laser facility, Technology, Manufacturing, Rapid Prototyping, FFF, FDM, 3D printing, Opto-Mechanical Design, HiLASE.

\section{Introduction}

HiLASE is a user scientific facility developing more than twenty laser systems for scientific and industrial applications, with output parameters ranging from a few picosecond pulses with energies of $5 \mathrm{~mJ}-0.5 \mathrm{~J}$ and repetition rates of $1-100 \mathrm{kHz}$ (thin disk technology) to systems with $100 \mathrm{~J}$ output energy in nanosecond pulses with a repetition rate of $10 \mathrm{~Hz}$ (multi-slab technology). [1] One of the key features of the HiLASE facility is its ability to deliver variety of these laser beams to experimental stations located in different user laboratory.

In the last few years, the use of additive technologies has found its way to all branches of industry. So it is no surprise that even the research institutions, like the HiLASE Centre, has decided to include this technology in its production processes. From the beginning, we did not expect such a good yield from implementing 3D printing technology at the HiLASE centre. But over time it proved to be the right move. The main advantage of the 3D printing technology is fast and cheap manufacturing of mostly good enough lightweight parts.

\section{Design Process}

At HiLASE, we use the FFF additive technology (Fused Filament Fabrication). This technology perfectly meets the needs of the HiLASE design team with its various versatility of production. Combining the development of the most powerful lasers in the world with the most basic 3D printing might seem strange at the first sight. But in the scope of time we have far succeeded to gain the most of benefits that FFF printing method offers.

\subsection{Boosting the Design Process}

The nature of a frontier technology research institute poses a lot of design challenges for the design team engineers. It happens frequently during the first stages of a design life-cycle that the initial design assignment and requirements changes several times so much, that it is easy to start from the scratch all over again.

In large scale, we combine the purchased components with the in-house made. Mostly due to lack of compatibility or lack of design features of the off-the shelf components we are driven to custom solutions. The design team has to usually implement and connect two and even more different systems to work together. This is the moment, when the 3D printing technology proves to be a very fast and effective tool that ease the design work.

\subsection{Eliminating the Idle Time}

By using 3D printing, we managed to significantly speed up the development process. Design parts, which were previously produced by the common subtractive methods, were delivered 4-6 weeks after the final approval of the design. Such long waiting times are very unfavourable for the development of new devices. All modifications take a lot of time. With the 3D print we are able to get hold of the parts within hours after design approval. So the parts can be assembled the second day after the completion of 
the 3D design. With such a high speed of prototype production, we are able to solve requests for design changes without major delays.

This gained flexibility proved very useful during user experiments at the HiLASE facility. Each user experiment is unique and therefore carefully planned several months in advance. If an initial analysis shows there is a compatibility error, e.g. wrong size of a sample holder, it is not possible to wait 4 weeks for a new one. In such cases, the 3D printing offers the instant solution. Within few hours, we are able to produce the necessary part and go on with preparation of the experiment.

The financial benefits of 3D printing cannot be overlooked either. The printed part is many times cheaper than a part produced by conventional methods. If we add the price for a delayed or cancelled experiment, the price difference would be higher by several orders. In the sum, whether we produce a part for $€ 100$ or $€ 10$ does not really matter, when the equipment time of device costing a million Euro is put on idle.

\section{Design Cases}

We will describe several custom design cases, which were solved with the 3D printing method.

\subsection{Connecting the Imperial with the Metric system}

The equipment used in the HiLASE Centre comes from all over the world. It is no exception that parts made in the USA, Germany and England are mounted together within a single laser device. The already mentioned design flexibility enables connecting different parts originally designed in Imperial and Metric systems together. Such a converting part fits the right dimensions of both systems (see Fig. 1 and Fig. 2). The printed parts can also be used to unify the threads. Sufficient accuracy and rigidity can be achieved by smart selection of the right material and proper print settings. By setting the shape and density of part infill we get components with tunable features.

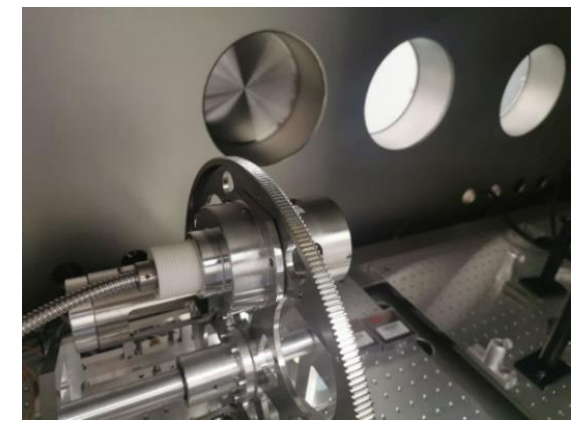

Figure 1. Threads unifier mounted in an experimental chamber

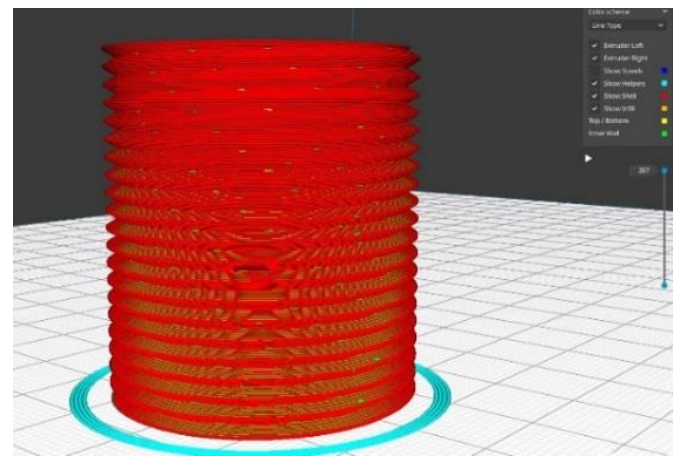

Figure 2. Sliced connector ready to be printed

\subsection{Rapid Prototyping}

The impact of manufacturing a complex shaped parts is marginal for an additive technology compared to a conventional production. Additive technologies allow exploring new ways of design.

For example we can easily combine materials within one part. See Fig. 3 for a part designed for optics polishing. This polishing head is made directly from two materials which results in better its better working performance. It is obvious that new parts, not limited by normal manufacturing processes, can be better adapted to their purpose.

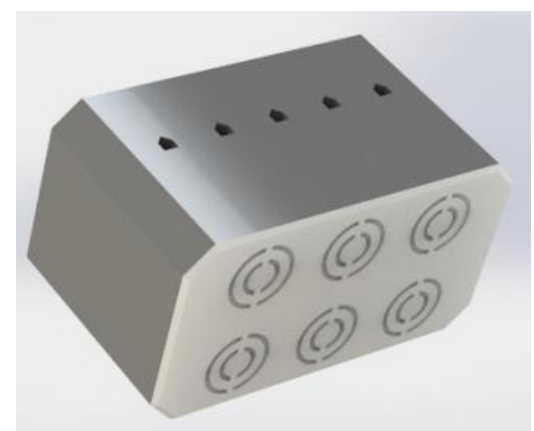

Figure 3. Two material composite tool for optics polishing

\subsection{Large Parts}

Optical components of the laser systems are sensitive to vibration. The opto-mechanical parts, stands and holders therefore have to be stiff. The performance stability of these laser sub-systems cannot be compromised by using plastic parts. [2] But still the 3D printing technology can be used as a first step in the design process to check the design viability. The complexity of our laboratory equipment and space limitation pose a not negligible risk of collision with other components. To avoid this risk, a cheap plastic dummy part is produced before ordering the production of an expensive part. This cheap dummy part is mounted to the system and thus we verify that the design is correct. If a collision occurs, the part is redesigned at less cost and time consumption. 


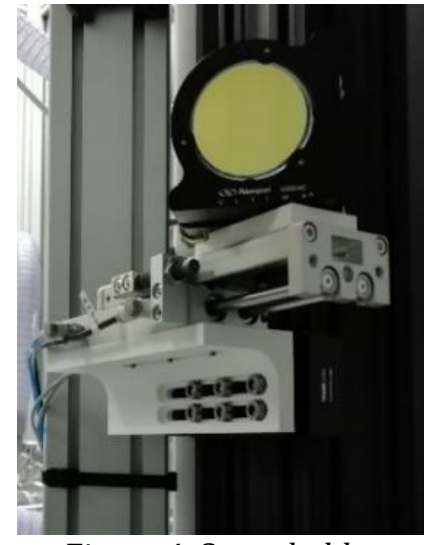

Figure 4. Stage holder

On Fig. 4 there is an example of a prototyped stage holder. It was designed on site in the laboratory according to imminent spatial restrains. It was printed and mounted within few days to keep a deadline for a user experiment. When the design concept proved its worth, the stage holder was manufactured from Aluminum and replaced the plastic one. The vibration stability of the plastic holder was lower than that made of metal but still good enough to meet the requirements.

\subsection{Vibration Attenuators}

We have a variety of practices to follow when designing a special use component. Apart from the bare design features, there is a large selection of materials to use with 3D printing method. We can choose a material that meets the mechanical and/or chemical requirements. The mechanical properties can be tuned by designing infill spatial structure of the part. We can print parts that significantly reduce the amount of transmitted vibration as well. We have already designed in-house rubber shock-absorbers and sealing. See Fig. 5 for one of these attenuators that is placed under a laser device.

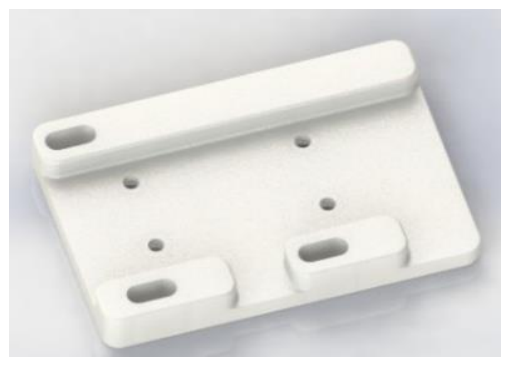

Figure 5. Rubber shock absorber

\subsection{Fiducials}

For optics alignment purposes we design our custom fiducials, which are applicable for different types and shapes of opto-mechanical holders. See Fig. 6 for a selection of samples. These can be produced in redundancy and help us save time during the assembly of laser systems.

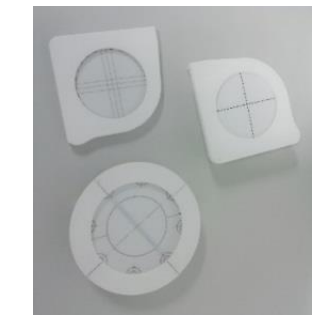

Figure 6. Adjustment tools

\section{Conclusion}

FFF is considered as future of manufacturing technology owing to its versatility and ability to convert an idea into real products at a faster pace as compared to other conventional processes. [3] Despite so many promising features, 3D printing method has limitations mostly originating from material properties. It still lacks in quality of surface finish and strength. Plastic material is generally not suitable for parts that require high rigidity and vibration stability. But by means of good design supported by smart print infill settings we can obtain very nice results.

We have started with one FFF printer and currently we have four. We bought two printers with multiple extruders, enabling the production of one part from multiple materials. By combining two materials, the part can acquire unique properties or different colors. The demand for printed parts is still growing and in the future it will be necessary to purchase additional equipment. The number of printed parts in 2019 exceeded 700 pieces. If everything continues at the same pace, we expect to reach the number of 1000 parts in 2020.

\section{Acknowledgement.}

This work was financed by the Ministry of Education, Youth and Sports of the Czech Republic (Programme NPU I Project No. L01602).

\section{References}

[1] Divoky, M., Smrz, M., Chyla, M., Sikocinski, P., Severova, P., Novak, O. et al., Overview of the HiLASE project: High average power pulsed DPSSL systems for research and industry, High Power Laser Science and Engineering 2. (2014). doi:10.1017/hpl.2014.16.

[2] Aliheidari N, Tripuraneni R., Hohimer C., Christ J., Ameli A., and Nadimpalli S. "The impact of nozzle and bed temperatures on the fracture resistance of FDM printed materials", Proc. SPIE 10165, Behavior and Mechanics of Multifunctional Materials and Composites 2017, 1016512 (11 April 2017); https://doi.org/10.1117/12.2260105.

[3] Shubham P., Sumit J.: Optimization of process parameter to improve dynamic mechanical properties of 3D printed ABS Polymer using Taguchi method, ARSSS International Conference, Goa (2018). 